\title{
A Case of Malignant PEComa of the Uterus Associated with Intramural Leiomyoma and Endometrial Carcinoma
}

\author{
Yoo Jin Choi · Jin Hwa Hong ${ }^{1}$ \\ Aeree Kim · Hankyeom Kim \\ Hyeyoon Chang \\ Departments of Pathology and ${ }^{1}$ Obsterics and \\ Gynecology, Korea University Guro Hospital, \\ Korea University College of Medicine, Seoul, \\ Korea \\ Received: February 12, 2016 \\ Revised: March 24, 2016 \\ Accepted: April 20, 2016 \\ Corresponding Author \\ Hyeyoon Chang, MD, PhD \\ Department of Pathology, Korea University Guro \\ Hospital, Korea University College of Medicine, \\ 148 Gurodong-ro, Guro-gu, Seoul 08308, Korea \\ Tel: +82-2-2626-3255 \\ Fax: +82-2-2626-1486 \\ E-mail: breakfree83@hanmail.net
}

\begin{abstract}
Perivascular epithelioid cell tumors (PEComas) refers to a family of mesenchymal neoplasms composed of angiomyolipomas, clear cell "sugar" tumors of the lung, and lymphangioleiomyomatoses. These tumors have a distinctive and common component of perivascular epithelioid cells that show an association with blood vessel walls and immunohistochemically display myomelanocytic differentiation. The unique neoplasms have been shown to have an expanded range through a variety of case reports, including visceral, intra-abdominal, soft tissue, and bone tumors. The retroperitoneum, abdominopelvic region, and uterus have been reported to be the most common sites. Most PEComas follow a benign course. However, reports of malignant PEComas are increasing. Many papers have described uterine PEComas, but to our knowledge, there have not yet been any reports of a malignant PEComa arising concomitant with another epithelial tumor and mesenchymal tumor. We report herein the case of a 67-year-old woman who experienced a malignant uterine PEComa infiltrating a preexisting intramural leiomyoma with synchronous well differentiated endometrial carcinoma and multiple liver and lung metastases.
\end{abstract}

Key Words: Immunihistochemistry; Perivascular epithelioid cell neoplasms; Uterus
Perivascular epithelioid cell tumors (PEComas) have a distinctive and common component of perivascular epithelioid cells that show an association with blood vessel walls and immunohistochemically display myomelanocytic differentiation. ${ }^{1-4}$ In this report, we present a unique case of a malignant uterine PEComa infiltrating a preexisting intramural leiomyoma with synchronous well differentiated endometrial carcinoma and multiple liver and lung metastases.

\section{CASE REPORT}

A 67-year-old post-menopausal woman visited the clinic due to vaginal bleeding. Ultrasonography revealed one myometrial mass and one endometrial lesion. Levels of serum tumor markers, including carbohydrate antigen 125 and carbohydrate antigen 19-9, were normal. Curettage of the endometrial lesion showed endometrioid adenocarcinoma, the International Federation of Gynecology and Obstetrics (FIGO) G1. Imaging studies revealed multiple suspicious metastatic lesions in the liver and lung. A total abdominal hysterectomy and bilateral salpingo-oophorec- tomy were performed.

There was an ill-demarcated grayish mass, measuring $4 \times 3.7$ $\times 3.5 \mathrm{~cm}$, on the anterior wall of the endometrium considered to be endometrial origin. There was another well-demarcated heterogeneous white to yellowish partially hemorrhagic and fibrotic mass, measuring $6 \times 5 \times 4.4 \mathrm{~cm}$, on the posterior wall of the myometrium. The cut surface of the mass showed an ill-demarcated yellowish necrotic lesion, enclosed by a whitish fibrotic area. It measured $3.7 \times 2.5 \times 2.5 \mathrm{~cm}$ (Fig. 1).

The endometrial lesion had the histologic appearance of endometrioid carcinoma, the same as the previous curettage (Fig. 2A). It invaded the myometrium $16 \mathrm{~mm}$ of the total $20 \mathrm{~mm}$ thickness.

The histologic findings of the myometrial mass were characterized by infiltration of spindle to ovoid cells into the surrounding leiomyoma. The infiltrating cells showed clear cytoplasm and elongated nuclei with cytological atypia. Frequent mitotic activity was encountered in up to 14/50 high-power fields (HPF). Coagulative tumor cell necrosis was present (Fig. 2B, C).

Immunohistochemical staining was performed on the myometrial lesion for $\mathrm{CD} 10$, caldesmon, desmin, $\alpha$-smooth muscle 


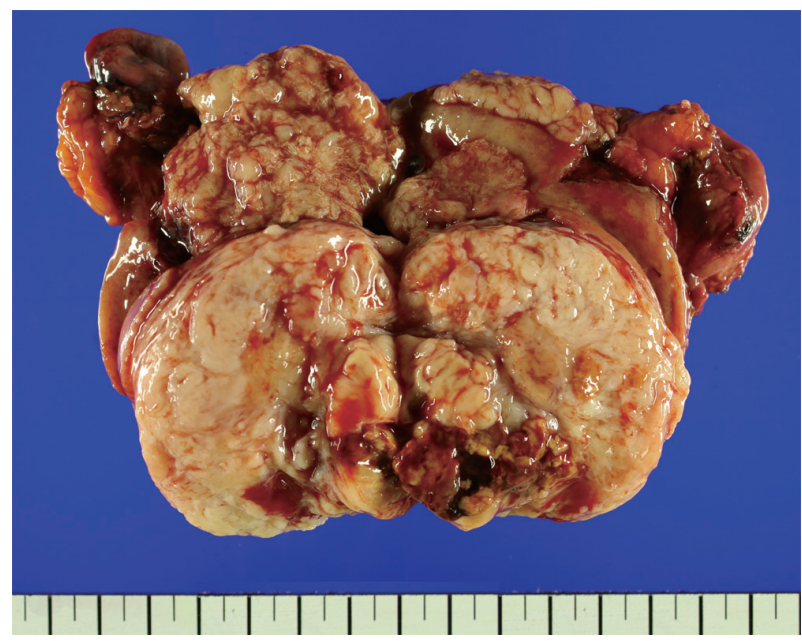

Fig. 1. Total hysterectomy showing endometriod carcinoma on anterior wall to fundus of the endometrium, and whitish leiomyoma encircling perivascular epithelioid cell tumors in the myometrium.

actin (SMA), human melanoma black 45 (HMB-45), Melan-A, transcription factor E3 (TFE3), and Ki-67. The infiltrating tumor cells were positive for SMA, HMB-45, and TFE3 with a low Ki-67 index of about 5\%, but they were negative for CD10, caldesmon, and desmin (Fig. 3A, B). In contrast, the surrounding leiomyoma showed positivity for caldesmon and desmin (Fig. 3C).

\section{DISCUSSION}

PEComas, which have no known normal tissue counterpart, are unique in that they react immunohistochemically for both melanocytic and myoid markers. ${ }^{2}$ Folpe et al. ${ }^{5}$ reviewed 26 cases of PEComas of soft tissue and gynecologic origin. According to that report, all cases demonstrated expression of at least one of the melanocytic markers (HMB-45, Melan-A, and microphthalmia transcription factor), and HMB- 45 showed the highest rate of positivity at $92 \%$. They also showed positivity for myoid markers with SMA (80\%) and desmin (36\%).

Our case demonstrated positivity for both HMB-45 and Melan$\mathrm{A}$, and melanin pigments were observed in some cells. Among the myoid markers, our case was positive for SMA and negative for desmin and caldesmon. The existing leiomyoma was positive for desmin and caldesmon, so the PEComa was clearly defined from the large preexisting leiomyoma at the peripheral portion of the bulky mass.

We reviewed all case series published in English available in PubMed that included more than three cases of PEComa in the female genital tract, for a total of 63 cases. The results (Table
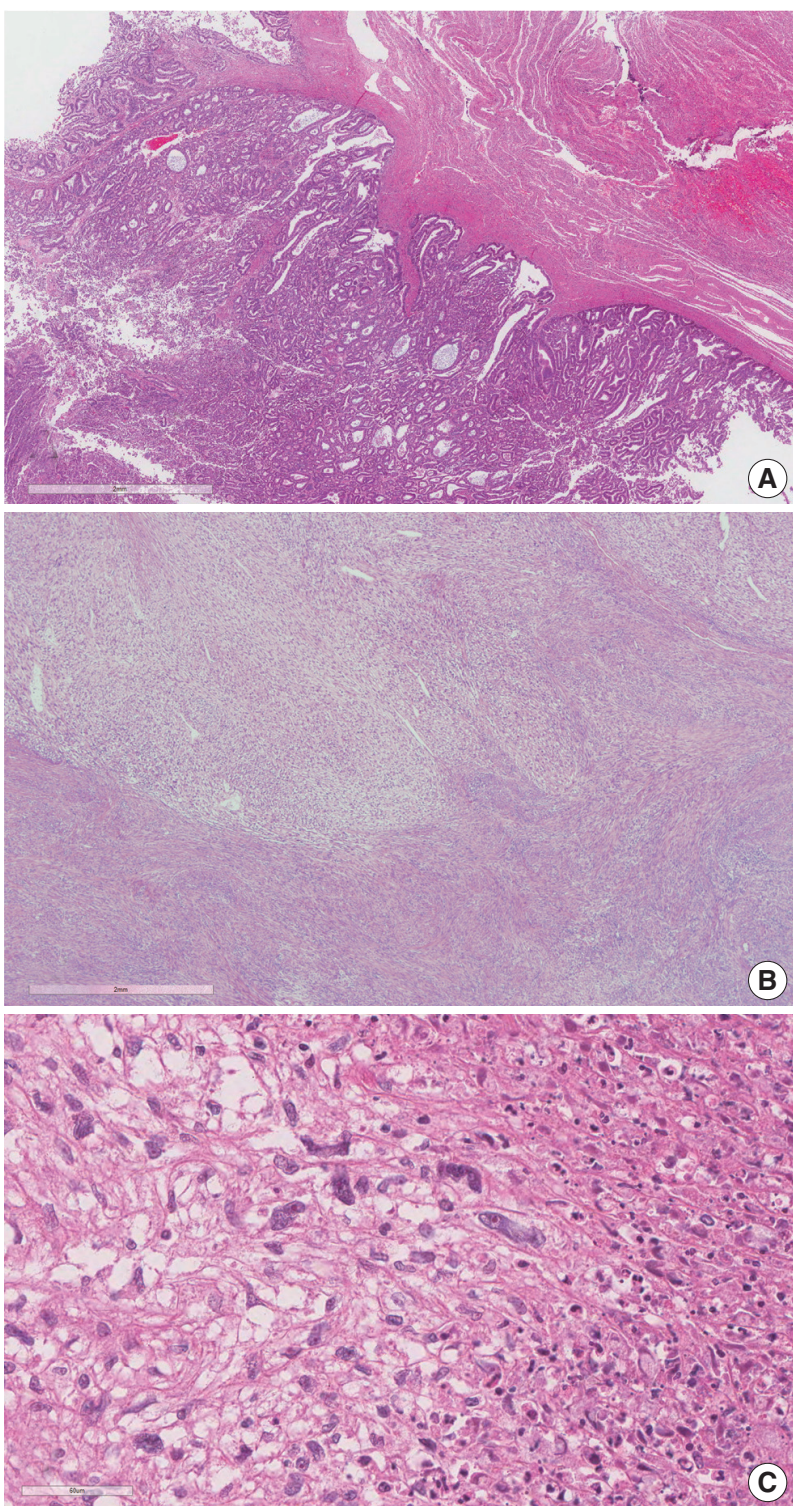

Fig. 2. (A) Endometrioid carcinoma, International Federation of Gynecology and Obstetrics (FIGO) G1. (B) Infiltrating spindle perivascular epithelioid cell tumor (PEComa) cells into surrounding leiomyoma. (C) The PEComa cells have elongated nuclei, prominent nucleoli, clear cytoplasm, cytological atypia, and necrosis.

$1)^{6-8}$ show that all (100\%) of the tested cases showed positivity for HMB-45, and 52.8\% were positive for Melan-A. Myoid markers including SMA, caldesmon, and desmin were positive in $74.5 \%, 66.0 \%$, and $94.4 \%$ of tested cases, respectively. CD10 immunostaining was performed in 23 cases and showed positivity in six cases (26.1\%). Five out of 13 cases (38.5\%) were positive for TFE3. These results confirm the diagnostic value of immunohistochemistry profiling in uterine PEComa, especially the use of melanocytic and myoid markers, due to their high rates of positivity. 


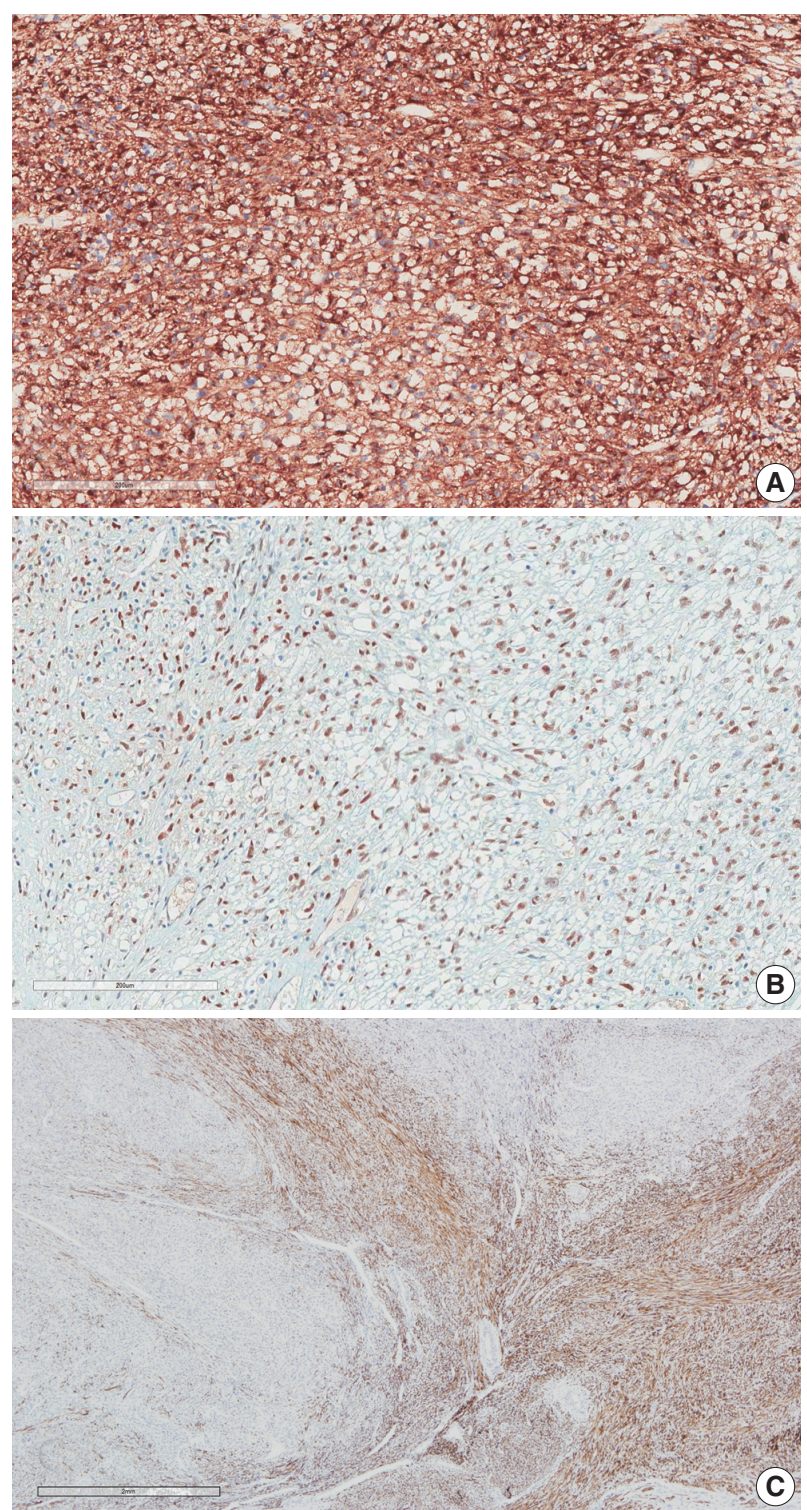

Fig. 3. (A) Human melanoma black 45 staining shows diffuse cytoplasm and membrane positivity. (B) Transcription factor E3 staining showed nuclear positivity. (C) Desmin stain is negative for perivascular epithelioid cell tumor and positive for leiomyoma. Infiltrative border is identified

The differential diagnosis of uterine PEComa includes uterine smooth muscle tumor, endometrial stromal tumor, gastrointestinal stromal tumor (GIST) with secondary involvement of the uterus, and other sarcomas such as alveolar soft part sarcoma (ASPS). Paraganglioma, metastatic melanoma/clear cell sarcoma of soft part, and rarely, carcinoma should also be considered in the differential diagnosis due to their epithelioid cytomorphology. Immunohistochemical staining may be helpful in the differential diagnosis. HMB-45 positivity enables a differential diagnosis of PEComa from uterine smooth muscle tumor, endometrial stromal

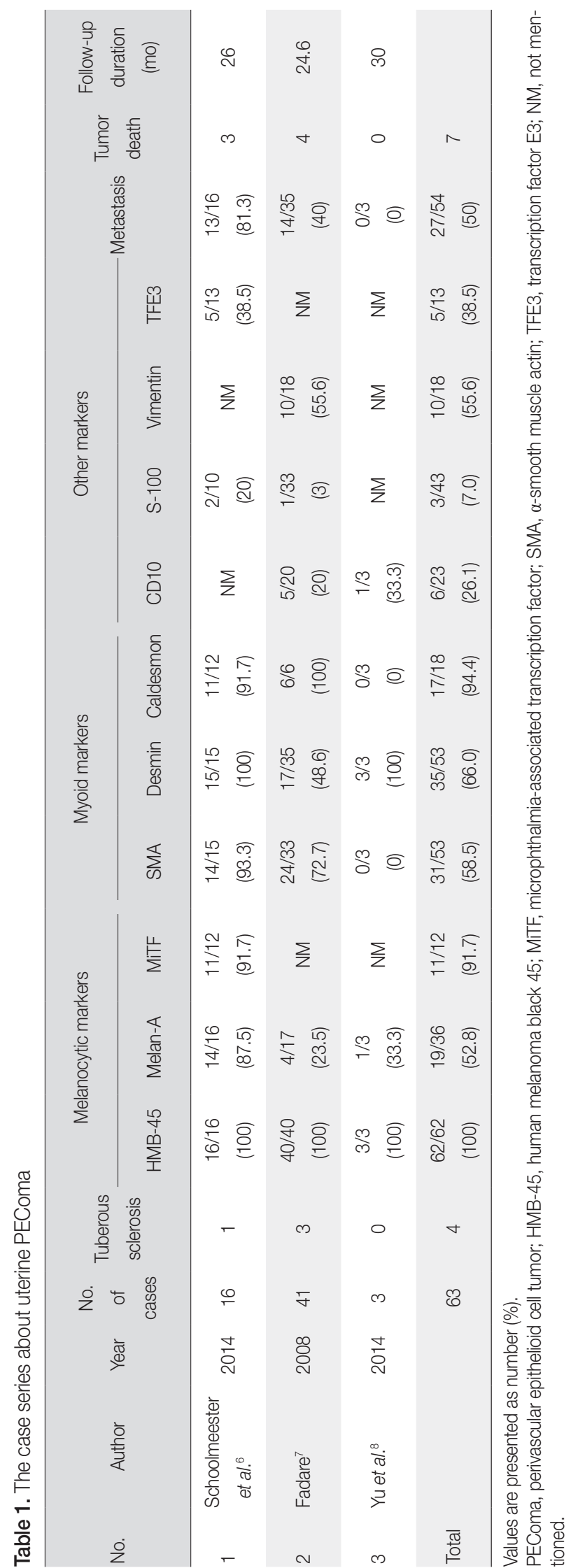


sarcoma, and ASPS. There was a report of late pulmonary and renal metastatic PEComas with initial misdiagnosis as uterine leiomyosarcomas. ${ }^{9} \mathrm{CD} 10$, which usually shows diffuse and strong immunoreactivity in endometrial stromal tumors, may be helpful in the differential diagnosis because $25 \%$ of uterine PEComas are reported to be positive for CD10. The exclusion of GIST from PEComa may be possible on the basis of $\mathrm{CD} 34$ staining, as well as c-Kit positivity and melanocytic marker negativity in GISTs. Metastatic melanoma and/or clear cell sarcoma can be distinguished from PEComa by the strong S-100 protein immunoreactivity of the former and their muscle marker negativity.

PEComas harboring TFE3 gene fusion are described in several reports. ${ }^{6,10,11}$ TFE3 is a member of the MiT family of transcription factors, and TFE3 gene fusions have been found in some neoplasms such as ASPS and a subset group of renal cell carcinoma. Our case also showed strong TFE3 immunoreactivity. In PEComa, the group harboring TFE3 gene fusion is reported to be more common in young patients, to be unrelated to tuberous sclerosis, to show alveolar architecture, and to have more epithelioid than spindle cell cytology and minimal immunoreactivity for myocytic markers. ${ }^{11}$ Because of the small number of cases, however, it is difficult to define these cases as a single distinct group showing specific clinical characteristics compared with conventional PEComas. More research and case studies are required.

There was a report of a concomitant PEComa and an endometrioid carcinoma with synchronous/metastatic bilateral ovary carcinomas and uterine leiomyoma. ${ }^{12}$ The case had a finding common to ours in that there was a well differentiated endometrial carcinoma involving a depth of more than half of the myometrium with a synchronous separate PEComa. In that case, however, the pathologic examination revealed benign features of PEComa and it presented as a subserosal mass, unlike our case, which presented as a protruding endometrial mass and showed malignant features.

Criteria for malignancy of PEComa of the female genital tract are currently not clearly defined due to insufficient case studies. According to criteria that are currently accepted, PEComas are classified into three categories: benign, uncertain malignant potential, and malignant. ${ }^{5,13}$ Benign is defined to exhibit the following gross or histologic features: gross size $<5 \mathrm{~cm}$, non-infiltrative growth, non-high-grade nuclear features, no necrosis or vascular invasion, and a mitotic rate $<1 / 50$ HPF. Tumors of uncertain malignant potential are defined as corresponding to one or more of the following features: nuclear pleomorphism or multinucleated giant cells, or gross size $>5 \mathrm{~cm}$ regardless of cellular features. Satisfying the malignant category refers to cases which show two or more of the following features: gross size $>5$ $\mathrm{cm}$, infiltrative growth, high-grade nuclear features, necrosis, vascular invasion, or a mitotic index $\geq 1 / 50 \mathrm{HPF}$. According to the largest single series of PEComas of gynecologic origin, the current classification system was very specific and sensitive for the diagnosis of malignant PEComa in the gynecologic tract. ${ }^{6}$ The most common solid organ metastatic site according to the study was the lung. Multiple metastases were observed in the lung and liver in our case.

\section{Conflicts of Interest}

No potential conflict of interest relevant to this article was reported.

\section{REFERENCES}

1. Bonetti F, Pea M, Martignoni G, et al. Clear cell ("sugar") tumor of the lung is a lesion strictly related to angiomyolipoma--the concept of a family of lesions characterized by the presence of the perivascular epithelioid cells (PEC). Pathology 1994; 26: 230-6.

2. Fletcher CD, Bridge JA, Hogendoorn P, Mertens F. WHO classification of tumours of soft tissue and bone. 4th ed. Geneva: World Health Organization, 2013.

3. Bonetti F, Martignoni G, Colato C, et al. Abdominopelvic sarcoma of perivascular epithelioid cells: report of four cases in young women, one with tuberous sclerosis. Mod Pathol 2001; 14: 563-8.

4. Folpe AL, Goodman ZD, Ishak KG, et al. Clear cell myomelanocytic tumor of the falciform ligament/ligamentum teres: a novel member of the perivascular epithelioid clear cell family of tumors with a predilection for children and young adults. Am J Surg Pathol 2000; 24: $1239-46$.

5. Folpe AL, Mentzel T, Lehr HA, Fisher C, Balzer BL, Weiss SW. Perivascular epithelioid cell neoplasms of soft tissue and gynecologic origin: a clinicopathologic study of 26 cases and review of the literature. Am J Surg Pathol 2005; 29: 1558-75.

6. Schoolmeester JK, Howitt BE, Hirsch MS, Dal Cin P, Quade BJ, Nucci MR. Perivascular epithelioid cell neoplasm (PEComa) of the gynecologic tract: clinicopathologic and immunohistochemical characterization of 16 cases. Am J Surg Pathol 2014; 38: 176-88.

7. Fadare O. Perivascular epithelioid cell tumor (PEComa) of the uterus: an outcome-based clinicopathologic analysis of 41 reported cases. Adv Anat Pathol 2008; 15: 63-75.

8. Yu Y, Shi HY, Huang HF. Uterine perivascular epithelioid cell tumour. J Obstet Gynaecol 2014; 34: 519-22.

9. Armah HB, Parwani AV. Malignant perivascular epithelioid cell tu- 
mor (PEComa) of the uterus with late renal and pulmonary metastases: a case report with review of the literature. Diagn Pathol 2007; 2: 45 .

10. Argani P, Aulmann S, Illei PB, et al. A distinctive subset of PEComas harbors TFE3 gene fusions. Am J Surg Pathol 2010; 34: 1395-406.

11. Malinowska I, Kwiatkowski DJ, Weiss S, Martignoni G, Netto G, Argani P. Perivascular epithelioid cell tumors (PEComas) harboring TFE3 gene rearrangements lack the TSC2 alterations characteristic of conventional PEComas: further evidence for a biological distinction. Am J Surg Pathol 2012; 36: 783-4.

12. Gao Z, Bhuiya T, Anderson A. Perivascular epithelioid cell tumour (PEComa) of the uterus associated with malignant neoplasm of the female genital tract. J Obstet Gynaecol 2004; 24: 600-4.

13. Folpe AL, Kwiatkowski DJ. Perivascular epithelioid cell neoplasms: pathology and pathogenesis. Hum Pathol 2010; 41: 1-15. 